\title{
Immune cell function assays in the diagnosis of infection in pediatric liver transplantation: an open-labeled, two center prospective cohort study
}

\author{
Feng Xue ${ }^{1 \#}$, Wei Gao ${ }^{2 \#}$, Tian Qin ${ }^{1}$, Cheng Wu ${ }^{3}$, Yi Luo ${ }^{1}$, Jing Chen ${ }^{2}$, Tao Zhou ${ }^{1}$, Mingxuan Feng ${ }^{1}$, \\ Bijun Qiu ${ }^{1}$, Jianjun Zhu ${ }^{1}$, Jia He ${ }^{3}$, Qiang Xia ${ }^{1,4}$
}

${ }^{1}$ Department of Liver Surgery and Liver Transplantation, Renji Hospital, School of Medicine, Shanghai Jiao Tong University, Shanghai, China;

${ }^{2}$ Transplant Center, Tianjin First Central Hospital, Tianjin, China; ${ }^{3}$ Department of Health Statistics, Second Military Medical University, Shanghai, China; ${ }^{4}$ Clinical Research Center, Shanghai Jiao Tong University School of Medicine, Shanghai, China

Contributions: (I) Conception and design: F Xue, W Gao, T Qin, Q Xia; (II) Administrative support: F Xue, W Gao, Q Xia; (III) Provision of study materials or patients: F Xue, W Gao, Q Xia; (IV) Collection and assembly of data: F Xue, W Gao, T Qin, Y Luo, J Chen, T Zhou, M Feng, B Qiu, J Zhu; (V) Data analysis and interpretation: F Xue, W Gao, Ti Qin, C Wu, J He, Q Xia; (VI) Manuscript writing: All authors; (VII) Final approval of manuscript: All authors.

\#These authors contributed equally to this work.

Correspondence to: Qiang Xia. Department of Liver Surgery and Liver Transplantation, Shanghai Jiao Tong University School of Medicine Ren Ji Hospital, No. 160 Pujian Road, Shanghai 200127, China. Email: xiaqiang@shsmu.edu.cn.

Background: Limited studies have been performed in assessment of immune status of pediatric liver transplants (PLTs). We conducted this study to evaluate Cylex immune cell function assay in diagnosis of infection and its potential clinical application in Chinese infant PLTs.

Methods: In this prospective cohort study, 227 infant PLTs from two medical centers were enrolled, and 216 completed the study. Cylex ATP values were measured before and after liver transplantation (LT) at week 1, 2, 3, 4, 8, 12 and 24 respectively. Accordingly, patients' clinical records, including demographic data, liver function results, tacrolimus dosages and concentrations were collected and analyzed.

Results: One hundred and sixty of 216 PLTs $(74.1 \%)$ were diagnosed infection based on the parameters including abnormal vital signs, imaging changes, and pathogens detection, while 44 (20.4\%) were clinically stable and 12 (5.6\%) experienced acute rejection. The median Cylex ATP value in infant PLTs post-surgery reduced significantly in infection group compared to stable group (median, $137 v s .269 \mathrm{ng} / \mathrm{mL}, \mathrm{P}<0.001$ ). Receiver operating characteristic (ROC) curve analysis determined that the cut-off value of Cylex ATP was $152 \mathrm{ng} / \mathrm{mL}$ in diagnosis of infection [area under the curve (AUC): 0.784, 95\% CI: 0.720-0.848]. Meanwhile, Cylex ATP value showed no correlation to tacrolimus dosage, blood concentration, dose-normalized concentration/dose ratio or Kaup index. However, it tended to correlate weakly with the white blood cell (WBC) number $(\mathrm{R}=0.462, \mathrm{P}<0.0001)$ and lymphocyte counts $(\mathrm{R}=0.363, \mathrm{P}<0.0001)$.

Conclusions: In this study, we demonstrated that low Cylex ATP represented partly overimmunosuppression and had diagnostic value in infant PLTs with infections, which might assist individualized immunosuppression in PLT patients.

Keywords: ImmuKnow assay; Cylex ATP; pediatric liver transplantation; infection

Submitted Aug 20, 2020. Accepted for publication Dec 23, 2020.

doi: $10.21037 /$ tp-20-256

View this article at: http://dx.doi.org/10.21037/tp-20-256 


\section{Introduction}

Liver transplantation (LT) is the only effective treatment for patients with end-stage liver disease, both adults and children (1-3). Immunosuppressive agent monitoring by trough level is the most commonly used regimen to evaluate post-transplant immune status; however, this approach does not provide an accurate reflection of the real-time immune status of the patient or adverse effects of the drug $(4,5)$. Moreover, a 10-fold variability in immunosuppressive pharmacokinetics and blood concentration have been observed after a fixed dose administration in pediatric recipients $(6,7)$. Based on measurement of peripheral $\mathrm{CD}^{+}$ $\mathrm{T}$ cell adenosine triphosphate (ATP) activity, the Cylex Immune Cell Function (ImmuKnow) Assay has been used to provide an overview of the immune response to transplant recipients receiving immunosuppressive therapy (8). However, a child immune system is in a developmental stage (9) and his immune cell composition differs from that of an adult (10); therefore, whether the Cylex ATP is capable of evaluating immune status in pediatric recipients or not is unclear. Currently, a normal range of Cylex ATP values have been established in healthy adult volunteers (225 to $525 \mathrm{ng} / \mathrm{mL}$ ) (11). However, some studies have shown that pediatric patients have lower values than those of adult patients, but yet has any results in infant patients $(12,13)$. Thus, the application of Cylex ATP assays in assessment of pediatric transplant patient, especially the infant recipient remains to be established.

Previous studies have proved that high Cylex ATPs are related to inadequate immunosuppression and high risk of rejection (14-16), while the low values represent excessive immunosuppression and increased risk of infection (17). The immunosuppressive dosage in children is usually much higher than that in adults probably due to a larger graft-recipient weight ratio (GRWR) and immature cytochrome P450 enzyme activity during the first year of life $(18,19)$. Compared to adults, children are at higher risk of infection and rejection after organ transplantation, up to now, infection is still a major cause of post-transplant death in children (20). Transplantation-related infections, especially opportunistic viral and fungal infections, are often associated with excessive immunosuppression, resulting in an extremely high mortality rate in children (21). However, despite individualized medication adjustment in responding to drug trough levels, there is still a lack of effective means to reflect the actual immunosuppression status. Therefore, identification of parameters that can reflect the immune status more accurately for young pediatric recipient under immunosuppression is of utmost importance for their proper drug dosage.

In this study, we analyzed the dynamic changes of Cylex ATP values in Chinese infant pediatric liver transplant (PLT) recipients before and post-transplantation, and evaluated the potential application of Cylex ATP assay in diagnosing transplant-associated infection in young pediatric patients.

We present the following article in accordance with the STROBE reporting checklist (available at http://dx.doi. org/10.21037/tp-20-256).

\section{Methods}

\section{Study design}

In this prospective cohort study, we applied ImmuKnow ATP assays in PLT recipients which are enrolled in two different medical centers (Shanghai Jiao Tong University School of Medicine Affiliated Renji Hospital and Tianjin First Center Hospital, China) between April 2017 and June 2018 to monitor their peripheral $\mathrm{CD}^{+} \mathrm{T}$ cell ImmuKnow ATP activity and investigate its potential to evaluate the diagnosis value in infection and reflect the actual immune status post-surgery. The study was registered in Chinese Clinical Trail Registry Center (No. ChiCTR1800015089).

The inclusion criteria of this study were: (I) patients aged $<3$ years at the time of transplantation; (II) patients who are ethnically Chinese; (III) parents or legal guardians have been informed of the enrolment and signed the consent.

The exclusion criteria were: (I) patients who have received medication that may have an effect on the pharmacokinetics of tacrolimus (TAC); (II) patients who received combined liver and kidney transplantations or secondary LTs; (III) patients who have been involved in other medical research in the 4 weeks prior to enrolment.

The Cylex ATP assay tests were scheduled at the following eight time-points: maximum of 5 days before transplantation and 1, 2, 3, 4, 8, 12, 24 weeks after surgery. We also recorded each recipient's vital signs, growth development parameters and detailed clinical data at each time-point. In addition to the time-point mentioned above, an extra test would be performed if an episode of infection or rejection or any other disease-related situation happened.

The following baseline data were recorded: the date of LT, sex, age, primary diagnosis of recipient, pediatric endstage liver disease (PELD) score, Child-Pugh score, results of routine blood examination and liver/kidney function. 
Moreover, dose and concentration of immunosuppressant drugs and other simultaneous medication were recorded, as well as pathology and imaging data, if available. All liver biopsies were evaluated independently by two or three experienced pathologists.

\section{Trial endpoint}

The clinical status of patients was categorized as: stable, infection, and rejection. Infection and rejection episodes were trial endpoints. Stable status was defined as normal clinical experimental and imaging examinations without infection or rejection. If the patients have occurred endpoint episodes, we would stop the research trial of ATP assay. While all patients would be followed up for clinical status and treatment during the trial.

Diagnosis of transplant-related infection was based on vital signs (for example, elevated body temperature, accelerated heart rate and breathing), hemogram changes (increased leucocytes and neutrophils) and imaging changes by chest radiography or computed tomography (CT) scan. In addition, the more accurate diagnosis would be the culture result of blood/sputum/abdominal fluid/jugular vein tube. C-reactive protein (CRP) and procalcitonin (PCT) tests would also be taken into consideration. Infection events were subdivided into bacterial, fungal and viral infections and rare pathogen infection. Fungal infections were indicated by $\beta 1,3$-glucanase positive, or culture positive, in addition to effective antifungal drug therapy. The viral infections consisted mainly of influenza virus, cytomegalovirus (CMV) and Epstein-Barr virus (EBV). The recipients were routinely screened by blood polymerase chain reaction (PCR) for $\mathrm{CMV}$ and $\mathrm{EBV}$, with positive results for viremia $\geq 400$ copies $/ \mathrm{mL}$. If the infection episodes got even severe to turn to sepsis, and the diagnosis of sepsis: a heart rate $>180$ or $<90 \mathrm{bpm}$, breathing $>34 \mathrm{bpm}$, white blood cell $(\mathrm{WBC})>17.5 \times 10^{9} / \mathrm{L}$ or $<5 \times 10^{9} / \mathrm{L}$ or systolic pressure $<100 \mathrm{mmHg}$ if the patients $<1$ year old; a heart rate $>140 \mathrm{bpm}$, breathing $>22 \mathrm{bpm}, \mathrm{WBC}>15.5 \times 10^{9} / \mathrm{L}$ or $<5 \times 10^{9} / \mathrm{L}$ or systolic pressure $<94 \mathrm{mmHg}$ if the patients are between 1 to 3 years old.

Rejection was defined by the results of liver function tests or pathological biopsy analysis. A clinical acute rejection was diagnosed as elevated liver enzyme values and continuously low blood trough concentration of TAC or cyclosporine (CsA), with liver function improved by additional dose of drugs. A pediatrician and a surgeon verified the diagnosis of post-operative infection or acute cellular rejection. The biopsy of liver tissue was considered to be the gold standard of acute cellular rejection.

\section{Patients and ethics statement}

This study was approved independently by the ethics committees of the two study centers approval No: [2016]134K and 2016033 KY\}. The study protocol conformed to the ethical guidelines of the Declaration of Helsinki (as revised in 2013). The immune function data from our study would not be used for clinical decisions.

\section{Immunosuppressive protocol}

An immunosuppressive protocol consisting of TAC and steroids was routinely applied postoperatively, with or without mycophenolate mofetil. TAC was started at day 2 or 3 after pediatric transplantation and the initial dose was $0.15 \mathrm{mg} / \mathrm{kg} /$ day orally, twice daily.

The dose was then adjusted according to blood trough concentration measured $12 \mathrm{~h}$ after the previous dose (termed $\mathrm{C} 0$ ). The target whole blood trough concentration of TAC (C0) was $8-12 \mathrm{ng} / \mathrm{mL}$ for the $1^{\text {st }}$ month, $7-10 \mathrm{ng} / \mathrm{mL}$ during the $2^{\text {nd }}-6^{\text {th }}$ months and $5-8 \mathrm{ng} / \mathrm{mL}$ thereafter. Of blood tests at each time-point, C0 was measured by a microparticulate enzyme immunoassay (IMx or i1000, Abbott Co., Ltd., Tokyo, Japan) according to the manufacturer's instructions. The weight-adjusted dosage $(\mathrm{mg} / \mathrm{kg} /$ day $)$ was calculated to give a dose-normalized concentration $\mathrm{C} / \mathrm{D}$ ratio, which was used to estimate the drug clearance.

\section{Cylex Immuknow assay}

Peripheral whole blood samples were collected into heparin anticoagulant tubes for Cylex ImmuKnow test (Inzex Biotechnology Company, Shanghai, China). Briefly, $250 \mu \mathrm{L}$ of sample was diluted with $750 \mu \mathrm{L}$ sample diluent, added to a 96-well microtiter plate and incubated overnight (16-18 h) with phytohemagglutinin stimulation at $37^{\circ} \mathrm{C}$ under $5 \%$ $\mathrm{CO}_{2}$. Anti-CD4 monoclonal antibody coated beads were added to the sample next day. The beads selected cells were lysed to release intracellular ATP, which was measured in a luciferin/luciferase assay using a luminometer, with the amount of light produced being proportional to the concentration of ATP $(\mathrm{ng} / \mathrm{mL})$. 


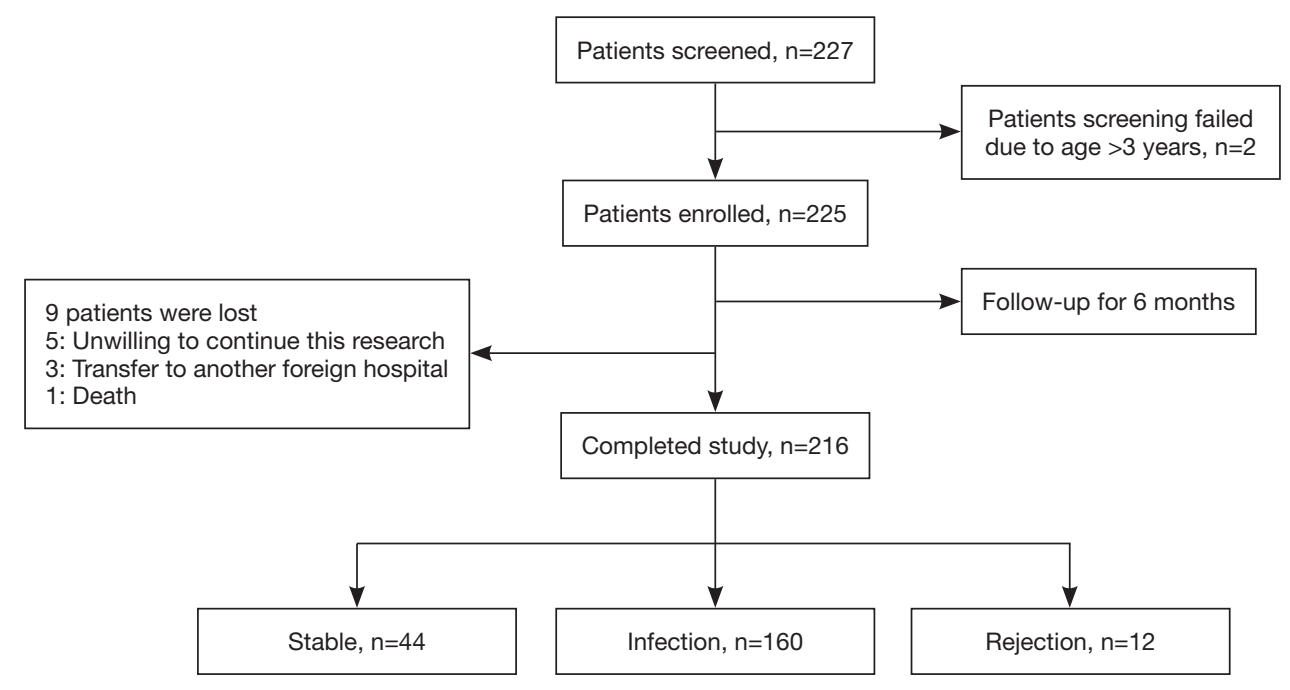

Figure 1 Flow chart of periods of recruitment, follow-up and data collection of the trial.

\section{Statistical analysis}

Continuous data were described as mean \pm standard deviation (SD) or median with interquartile range (IQR). Qualitative data were described as percentages (\%). The trend analysis of ATP over time was performed using repeated measures analysis of variance (ANOVA). For continuous data, we first used Kolmogorov-Smirnov test to test the normality and homogeneity of variance. If assumed, ANOVA was used, if not assumed, Kruskal-Wallis rank sum test was used to compare three groups. For categorical data, chi-square test was used to compare three groups. To compare ATP of patients with different outcomes, we used Kruskal-Wallis rank sum test at first, then Wilcoxon rank sum test to make multiple comparisons. In multiple comparisons, we adjusted $\alpha$ as 0.025 to avoid increasing type I error. All tests were two-side test. Correlations of WBC counts, neutrophil counts, lymphocyte counts, monocyte counts, TAC trough concentration and dosenormalized concentration (C/D ratio) with the Immuknow ATP values were tested by Pearson's correlation analysis. Receiver operating characteristic (ROC) curves were used to evaluate the performance of Cylex Immuknow ATP in assessing the clinical status of pediatric patients. For recipients who developed rejection or infection events, the ATP value when the event occurred was used for statistical analysis; for patients who did not experience such events, the ATP values at post-operative week 4 were applied to reflect the stable immune situation. The area under the curve (AUC) and 95\% CI, cut-off point, sensitivity and specificity were calculated. A P value $<0.05$ was considered to indicate statistical significance. All statistical analyses were performed with SPSS, version 23.0. Graphical plots were generated using GraphPad Prism 6.0 (GraphPad Software, La Jolla, CA, USA).

\section{Results}

\section{Patients' clinical characteristics}

Two hundred and sixteen (111 male) of 227 PLT recipients had finished the study, while two children failed screen due to age over 3 years, nine recipients loss to follow-up during observation ( 5 cases: lost interest, 3 cases: moved, 1 case: death) in the study between April and June 2017. Overall survival rate was $99.5 \%(215 / 216)$, expect for one died of suffocation due to vomiting at October, 2017. Figure 1 showed the complete flow chart of the study. Table 1 showed the descriptive data of the enrolled recipients. In short, children's median age at transplantation was 7 months (range, 3-36 months) and $85.6 \%(\mathrm{n}=185)$ were less than 1 year old. Their median weight was $7.0 \mathrm{~kg}$ (range, 4.6-16.0 kg). The most indication was biliary atresia $(\mathrm{n}=201,93.1 \%)$, followed by congenital hepatic fibrosis $(n=7)$, Alagille syndrome $(n=3)$, Caroli disease $(n=1)$, methylmalonic academia $(n=3)$ and ornithine transcarbamylase deficiency $(\mathrm{n}=1)$, respectively. Among the PLT recipients, 98.1\% ( $\mathrm{n}=211,97.7 \%)$ underwent living donor LT (LDLT), while the others underwent deceased donor LT (DDLT). The median PELD score was 13.0 (range, -6 to 45 ). Patients 
Table 1 Characteristics of the enrolled pediatric transplant recipients

\begin{tabular}{|c|c|c|c|c|c|}
\hline Characteristics & $\begin{array}{l}\text { Data of recipients } \\
\qquad(n=216)\end{array}$ & $\begin{array}{l}\text { Stable group } \\
\qquad(n=44)\end{array}$ & $\begin{array}{l}\text { Infection group } \\
\qquad(n=160)\end{array}$ & $\begin{array}{l}\text { Rejection group } \\
\qquad(\mathrm{n}=12)\end{array}$ & $P$ value \\
\hline Age (months), median (IQR) & $7.0(4.0)$ & $7.0(3.8)$ & $7.0(3.0)$ & $9.5(6.3)$ & 0.266 \\
\hline Male & $111(51.4)$ & $24(54.5)$ & $80(50.0)$ & 7 (58.3) & \\
\hline Height (cm), median (IQR) & $66.0(6.8)$ & $64.5(6.8)$ & $66.0(6.0)$ & $66.5(4.8)$ & 0.333 \\
\hline Type of transplant, $\mathrm{n}(\%)$ & & & & & 0.218 \\
\hline DDLT & $5(2.3)$ & $0(0)$ & $5(3.1)$ & 0 & \\
\hline $\mathrm{CHF}$ & $7(3.2)$ & $1(2.3)$ & $6(3.8)$ & 0 & \\
\hline Alagille syndrome & $3(1.4)$ & 0 & $3(1.9)$ & 0 & \\
\hline Caroli disease & $1(0.5)$ & 0 & $1(0.6)$ & 0 & \\
\hline MA & $3(1.4)$ & $1(2.3)$ & $2(1.3)$ & 0 & \\
\hline OTCD & $1(0.5)$ & 0 & $1(0.6)$ & 0 & \\
\hline Child-Pugh classification, n (\%) & & & & & 0.300 \\
\hline$A$ & $17(7.9)$ & $5(11.4)$ & $12(7.5)$ & 0 & \\
\hline
\end{tabular}

IQR, interquartile range; LDLT living donor liver transplantation; DDLT, deceased donor liver transplantation; CHF, congenital hepatic fibrosis; MA, methylmalonic academia; OTCD, ornithine transcarbamylase deficiency; PELD, pediatric end-stage liver disease; GRWR, graft to recipient weight ratio.

were categorized as Child-Pugh A, B, C in 7.9\% ( $\mathrm{n}=17)$, $65.3 \%(n=141)$ and $26.9 \%(n=58)$ of cases, respectively.

During the whole study period, infection took the major part of adverse event and occurred in $74.1 \%(\mathrm{n}=160)$ of patients, while $20.4 \%(n=44)$ of PLTs were clinically stable and rejection occurred in $5.6 \%(\mathrm{n}=12)$. No significant difference was found in the characteristic parameters among the three groups (Table 1). Post-transplant infection decreased from $27.3 \%$ to $12.1 \%$ during the first 4 weeks; and bacteria was the most infectious pathogen; however, the incidence of infection then increased two-fold at week 8 and kept at high levels till the end of the study, with $28.2 \%$ at week 24 (Figure 2), and EBV or CMV infection took the majority, accounting for $89.1 \%$ [49/55]. Besides, there were 38 episodes of clinical rejection, and only six were biopsyproven acute cellular rejection with Rejection Activity Index (RAI) $\geq 5$.

\section{Cylex ATP values correlated with clinical infection}

Totally we collected 1,431 blood samples for detection Cylex ATP according to the study schedule. To establish 


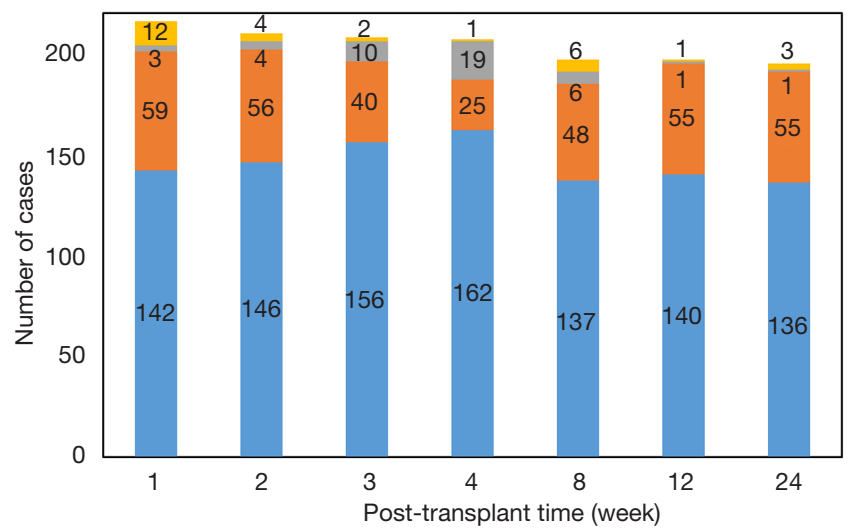

๓Stable $₫$ Infections $\llbracket$ Acute cellular rejection $\backsim$ Other adverse events

Figure 2 Composition of post-transplant immune status (stable, infection, acute cellular rejection and other transplant-related complications) in pediatric liver transplant recipients at each timepoint.

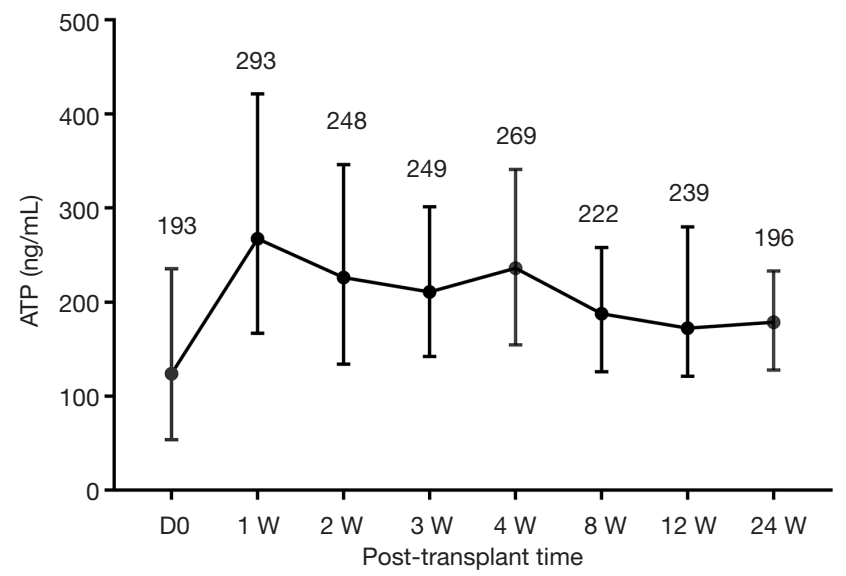

Figure 3 The distribution of ImmuKnow assay values at each time point from pre-transplant (D0) to post-transplant (1 W/2 W/3 $\mathrm{W} / 4 \mathrm{~W} / 8 \mathrm{~W} / 12 \mathrm{~W} / 24 \mathrm{~W}$ ). The results show was no significant impact of time on ImmuKnow values $(\mathrm{P}>0.05)$. W, week.

the detection system, we first analyzed Cylex ATP activities in clinically stable PLTs less than 3 years old dynamically to illustrate their detection features before and posttransplantation. The median ATP value was $193 \mathrm{ng} / \mathrm{mL}$ before LT, which then increased a little to an overall median level of $269 \mathrm{ng} / \mathrm{mL}$ in stable patients after LT, but there showed no significant difference at each detection points post-LT (Figure 3), indicating a stable immune status before and after LT for young recipients.
To investigate its potential value in evaluating immune status of PLT recipients, we compared the Cylex ATP values in different groups according to clinical diagnosis (infection, stable and rejection). Despite the similar baseline data in each group (Table 1), the median of Cylex ATP values were significantly lower in the infection group than those in the stable group (137 vs. $269 \mathrm{ng} / \mathrm{mL}, \mathrm{P}<0.0001$ ), but there was no difference between the rejection and stable groups (Figure 4A). Because median of the Cylex ATP values were lower in the infection group, we then performed ROC curve analysis to determine the sensitivity and specificity of the assay in the diagnosis of infection, and evaluated its potential application in identification of transplant-related infection. The ROC curve analysis showed the area under curve was 0.784 (95\% CI: $0.720-0.848, \mathrm{P}<0.0001)$ suggesting Cylex ATP assay had a medium performance in the diagnosis of infection in PLT recipients (Figure 4B). The maximum value Youden Index was 0.528 , and according to the threshold value for Youden Index is maximized, the optimal cut-off value of Cylex ATP in diagnosis of infection was $152 \mathrm{ng} / \mathrm{mL}$, at which diagnostic sensitivity and specificity were 0.573 and 0.955 , respectively.

\section{Correlation of Cylex ATP and other laboratory parameters}

Immunosuppressive drug dosage and concentration could reflect the strength of immunosuppression; therefore, we also compared the recorded data and drug usage between the stable and infection groups (Table 2). Due to few patients treated with CsA as an immunosuppressive drug, we used the number of patients treated with TAC in this analysis. There was no significant difference in either drug dosage or its concentration between these two groups.

We also analyzed the relationship between Cylex ATP and immunosuppressive drug administration post-LT. As shown in Figure 5, it didn't suggest there existed linear correlation between TAC concentration or TAC dosage and Cylex ATP values [Pearson correlation coefficients: $\mathrm{R}=0.063(\mathrm{P}=0.109)$ and $\mathrm{R}=-0.05(\mathrm{P}=0.18)$ respectively]. Moreover, since the pediatric nutrition status was related to the maturation of immune system, we also analyzed the correlation of nutritious index (Kaup) to Cylex ATP analysis, and did not indicate that Kaup index had linear correlation with Cylex ATP either. WBC and lymphocyte counts in peripheral blood could reflect the immune status partially, in our analysis, indeed, Cylex ATP levels tended to correlate weakly with the total WBC number $(\mathrm{R}=0.462$, $\mathrm{P}<0.0001)$ and lymphocyte counts $(\mathrm{R}=0.363, \mathrm{P}<0.0001)$. 
A

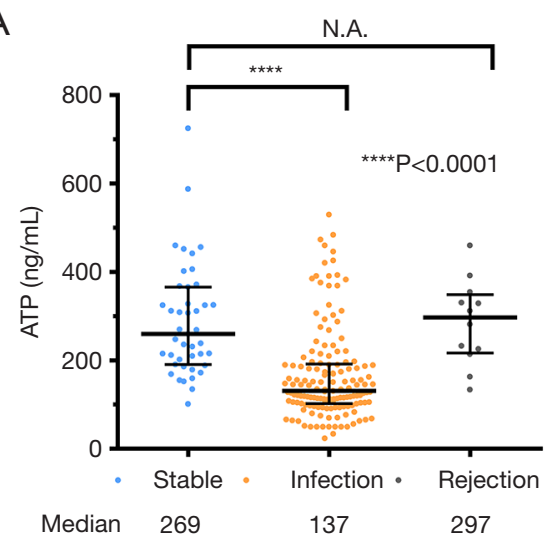

B

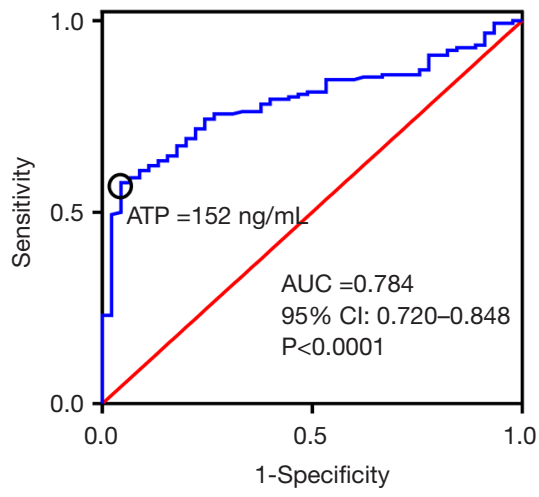

Figure 4 Analysis of ImmuKnow values in all groups. (A) ImmuKnow values in the infection group were significantly lower than those in stable group $(\mathrm{P}<0.0001)$. (B) The receiver operating characteristic $(\mathrm{ROC})$ curves of ImmuKnow values in predicting infection. The area under the curve (AUC) of the assay was 0.784 (95\% CI: 0.720-0.848). Youden Index had a maximum value when the ImmuKnow value was $152 \mathrm{ng} / \mathrm{mL}$, with sensitivity and specificity of 0.573 and 0.955 , respectively.

Table 2 Comparison of dose and concentration of tacrolimus between the stable and infection group

\begin{tabular}{|c|c|c|c|c|c|c|}
\hline Posttransplant time & \multicolumn{3}{|c|}{ Does of tacrolimus (mg/kg/d) } & \multicolumn{3}{|c|}{ Tacrolimus concentration ( $\mathrm{ng} / \mathrm{mL}$ ) } \\
\hline $1 \mathrm{~W}$ & $0.15 \pm 0.07$ & $0.14 \pm 0.06$ & & $8.28 \pm 2.91$ & $9.53 \pm 3.57$ & \\
\hline $2 \mathrm{~W}$ & $0.21 \pm 0.09$ & $0.19 \pm 0.08$ & & $8.19 \pm 3.68$ & $7.33 \pm 2.79$ & \\
\hline $3 \mathrm{~W}$ & $0.21 \pm 0.09$ & $0.19 \pm 0.08$ & & $7.27 \pm 2.67$ & $7.27 \pm 2.73$ & \\
\hline $8 W$ & $0.20 \pm 0.08$ & $0.21 \pm 0.10$ & & $8.01 \pm 3.21$ & $7.29 \pm 3.32$ & \\
\hline $12 \mathrm{~W}$ & $0.20 \pm 0.08$ & $0.20 \pm 0.11$ & & $6.96 \pm 1.90$ & $7.77 \pm 3.65$ & \\
\hline $24 \mathrm{~W}$ & $0.10 \pm 0.06$ & $0.14 \pm 0.09$ & & $6.55 \pm 1.77$ & $8.30 \pm 3.33$ & \\
\hline
\end{tabular}

W, week.

\section{Discussion}

Many studies have been conducted to evaluate the potential of the Cylex ATP Assay to predict the infection/ rejection events after organ transplantation (14,22-24). We characterized the Cylex ATP values in young PLT recipients at seven time points post-surgery to investigate its potential application. Moreover, we identified the cut-off point of the Cylex ATP value for diagnosis of liver transplant-associated infection in young PLTs.

Despite surgical developments in the field of transplantation, infectious complications remain a major cause of morbidity and mortality for pediatric patients after transplantation. Studies have showed that transplant patients who develop systemic infections could have a worse prognosis (25-27). In our study, it has been noted that infection rate reached $74.1 \%$, and infection cases took up the majority of adverse events. Concerning to the pathogens of infection dynamically, Infections occurred during 1 to 4 weeks post-surgery caused predominantly by bacteria and fungus, while asymptomatic viral infections occurred mainly during 8 to 24 weeks period. This phenomenon is in accordance with previous reports that most bacterial infections during the first month post-transplantation are related to a surgical procedure with medical complications. Opportunistic infections were predominantly viral infections occurring from the 2 to 6 months after LT, depending on risk factors and the intensity of immunosuppression, 
A

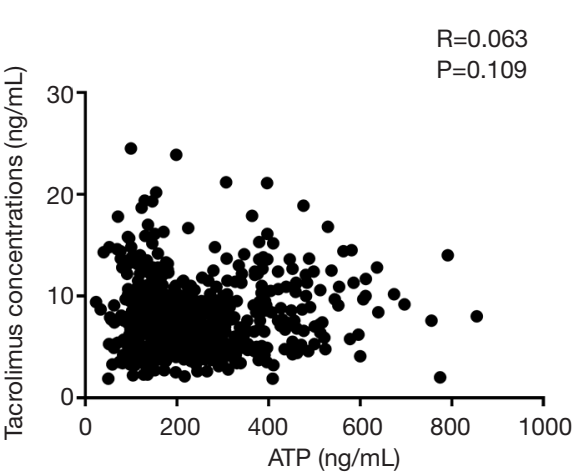

C

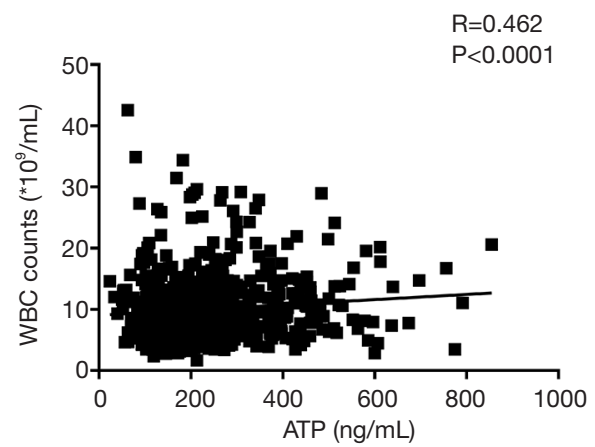

$E$

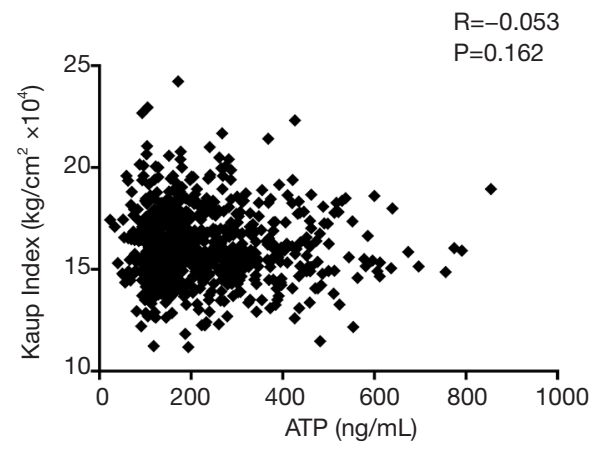

B

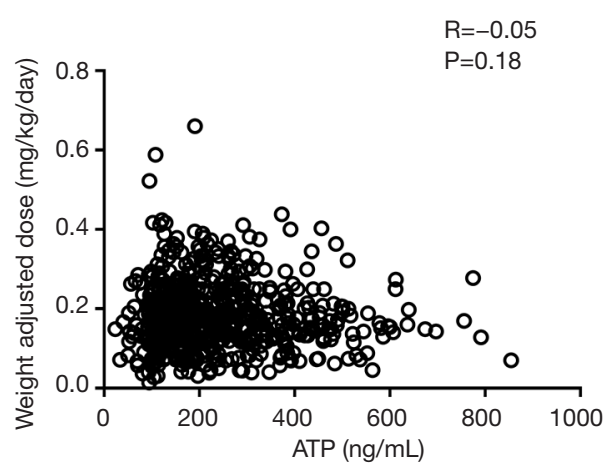

$\mathrm{D}$

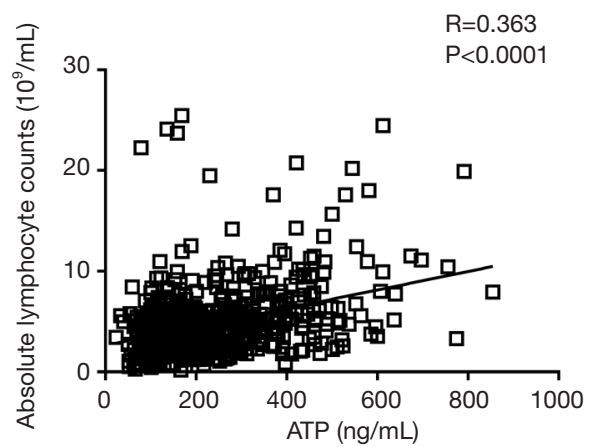

Figure 5 Correlation of ImmuKnow ATP levels with other clinical parameters, including: (A) TAC concentration; (B) TAC dosage; (C) white blood cell (WBC) counts; (D) lymphocyte numbers; and (E) Kaup index. Data show no correlations between ImmuKnow values and other clinical parameters. TAC, tacrolimus; C/D ratio, TAC trough concentration/dose-normalized concentration.

specifically over-immunosuppression (28). Previous studies have shown that CMV and/or EBV infection took place mainly between 30 to 90 days after transplantation and commonly in the first year after PLT. Most of the patients have viremia but remain asymptomatic, and EBV infection is a risk factor for the pathogenesis of post-transplantation lymphoproliferative disorder (PTLD) (21,29). To improve the long-term patient and graft survival, a more precise and individualized immunosuppressive drug regimen should consider decreasing the risk of infections at the same time.

Optimization of the immunosuppressive treatment for liver transplant recipients, especially for pediatric patients is still challenging. In this study with PLT recipients younger than 3 years old, these children have a quite different features in immune system compared to that of adults and teenagers $(30,31)$. However, few studies have focused on the immune response of young PLTs under immunosuppression, because there is very limited number of 
infantile PLTs in a single center. Here we enrolled 227 PLTs under 3 years old from two transplant centers to study this field intensively.

The characteristics of the immune response in young children are distinct from that of adults, and it is very difficult to judge their proper intensity of immunosuppression after LT. This is the first two-centered cohort study conducted in PLT recipients using Cylex ATP assay to evaluate the immune status in PLT less than 3 years old, our result show that median Cylex ATP is $269 \mathrm{ng} / \mathrm{mL}$ in stable PLT, which is much lower than that in our previous report in adult patients $(32,33)$. Our result is similar to some other reports that children have significantly lower Cylex ATP levels than those detected in adults (16,34).

In our study, we have determined that low Cylex ATP values correlated to transplant-related infection. The rate of asymptomatic viral infection in the study increased from 8 weeks post-surgery, meanwhile, Cylex ATP value gradually reduced accordingly, which indicated clinical over-immunosuppression in the PLT recipients, despite the fact that major of the pediatric patients had their TAC concentrations less than the recommended range, even be given at a higher dosage. Based on this information, we concluded that drug modification by TAC trough levels only might not be appropriate for monitoring PLT recipients because of the high risk of over-immunosuppression and leading to opportunistic viral infection. Thus, we proposed using Cylex ATP to assist in determination of personalized immunosuppressive administration in pediatric recipients. Besides, the assay is independent of immunosuppressive trough levels and is focus on the actual immune status post-LT, which may be in aid of trough monitoring to fulfill personalized immunosuppression in PLTs (35).

ROC analysis of Cylex ATP assay revealed the AUC of infection was 0.784 in the study, which represented a medium diagnostic value. A meta-analysis of adult solid organ transplantation by Ling et al. showed that the Cylex ATP had an AUC for infection of 0.77 (36), similar to our results. The results above imply that Cylex ATPs may have a potential application in evaluating over-immunosuppression and diagnosing transplant-associated infection. We still need further study to testify whether we take low Cylex ATP (less than $152 \mathrm{ng} / \mathrm{mL}$ ) into consideration of dosage adjustment will reduce infectious events or not.

Besides, acute rejection is another big problem after organ transplantation. However, we could not conclude high Cylex ATPs correlate to transplant rejection, because we have very limited cases or biopsy-proven rejection in the study. Nevertheless, some reports have also indicated that high Cylex ATP values do not correlate with graft rejection $(37,38)$. Besides, some studies have tried other biomarker to monitor clinical rejection, Ashokkumar et al. has applied a new measurement to use allo-specific CD $154^{+} \mathrm{T}$-cytotoxic memory cells to predict acute cellular rejection after liver or intestine transplantation in children (39), he believes CD154 T-cytotoxic memory is more precise in prediction of rejection than Cyelx ATPs.

However, some limitations of the study should be noted. First, because of the age limitation and the rare of primary disease, the study included a small sample size. Second, pediatric LT is a high surgical technique and be mastered as routine surgical only in a few centers in China at present. For the reason, this study was registered as a multiply-center trial containing only three independent medical centers, where had performed over $80 \%$ of total PLTs in China. However, there was no PLT suitable for enrollment from one center during the study and we finally had to assess the data from the other two centers. Third, despite the fact that low Cylex ATP correlated to transplant-associated infections and moderate diagnostic accuracy of ATP, we still need a further prospective randomized controlled trial (RCT) to verify whether it can represent over- immunosuppression and assist personalized immunosuppression clinically.

In summary, the results of our study have confirmed the diagnostic utility of the Cylex ATP assay in infection events after PLT. Thus, it offers the potential to aid in clinical decision-making on immunosuppressive drug usage to avoid the risk of infection, thereby may improve clinical outcomes.

\section{Acknowledgments}

Funding: This study was supported by Shanghai Municipal Hospital Three-year-project for Clinical Skills' Promotion and Innovation (16CR1003A); Shanghai Jiao Tong University School of Medicine (DLY201606); and National Natural Science Foundation of China (grant No. 81670602).

\section{Footnote}

Reporting Checklist: The authors have completed the STROBE reporting checklist. Available at http://dx.doi. org/10.21037/tp-20-256 
Data Sharing Statement: Available at http://dx.doi. org/10.21037/tp-20-256

Conflicts of Interest: All authors have completed the ICMJE uniform disclosure form (available at http://dx.doi. org/10.21037/tp-20-256). The authors have no conflicts of interest to declare.

Ethical Statement: The authors are accountable for all aspects of the work in ensuring that questions related to the accuracy or integrity of any part of the work are appropriately investigated and resolved. The study was conducted in accordance with the Declaration of Helsinki (as revised in 2013). The study was approved by institutional ethics board of the two study centers \{NO.: [2016]134K and $2016033 \mathrm{KY}\}$ and informed consent was taken from all the patients' parents.

Open Access Statement: This is an Open Access article distributed in accordance with the Creative Commons Attribution-NonCommercial-NoDerivs 4.0 International License (CC BY-NC-ND 4.0), which permits the noncommercial replication and distribution of the article with the strict proviso that no changes or edits are made and the original work is properly cited (including links to both the formal publication through the relevant DOI and the license). See: https://creativecommons.org/licenses/by-nc-nd/4.0/.

\section{References}

1. Murray JE. Human organ transplantation: background and consequences. Science 1992;256:1411-6.

2. Schuppan D, Afdhal NH. Liver cirrhosis. Lancet 2008;371:838-51.

3. Leonis MA, Balistreri WF. Evaluation and management of end-stage liver disease in children. Gastroenterology 2008;134:1741-51.

4. Wieland E, Olbricht CJ, Susal C, et al. Biomarkers as a tool for management of immunosuppression in transplant patients. Ther Drug Monit 2010;32:560-72.

5. Jalil MH, Hawwa AF, McKiernan PJ, et al. Population pharmacokinetic and pharmacogenetic analysis of tacrolimus in paediatric liver transplant patients. $\mathrm{Br} \mathrm{J}$ Clin Pharmacol 2014;77:130-40.

6. Prytuła AA, Cransberg K, Bouts AH, et al. The Effect of Weight and CYP3A5 Genotype on the Population Pharmacokinetics of Tacrolimus in Stable Paediatric
Renal Transplant Recipients. Clin Pharmacokinet 2016;55:1129-43.

7. Kassir N, Labbe L, Delaloye JR, et al. Population pharmacokinetics and Bayesian estimation of tacrolimus exposure in paediatric liver transplant recipients. $\mathrm{Br} \mathrm{J}$ Clin Pharmacol 2014;77:1051-63.

8. Zeevi A, Lunz J. Cylex ImmuKnow Cell Function Assay. Methods Mol Biol 2013;1034:343-51.

9. Ygberg S, Nilsson A. The developing immune system from foetus to toddler. Acta Paediatr 2012;101:120-7.

10. Saule P, Trauet J, Dutriez V, et al. Accumulation of memory $\mathrm{T}$ cells from childhood to old age: central and effector memory cells in CD4(+) versus effector memory and terminally differentiated memory cells in CD8(+) compartment. Mech Ageing Dev 2006;127:274-81.

11. Bhorade SM, Janata K, Vigneswaran WT, et al. Cylex ImmuKnow assay levels are lower in lung transplant recipients with infection. J Heart Lung Transplant 2008;27:990-4.

12. Israeli $M$, Klein T, Sredni B, et al. ImmuKnow: a new parameter in immune monitoring of pediatric liver transplantation recipients. Liver Transpl 2008;14:893-8.

13. Hooper E, Hawkins DM, Kowalski RJ, et al. Establishing pediatric immune response zones using the Cylex ImmuKnow assay. Clin Transplant 2005;19:834-9.

14. He J, Li Y, Zhang H, et al. Immune function assay (ImmuKnow) as a predictor of allograft rejection and infection in kidney transplantation. Clin Transplant 2013;27:E351-8.

15. Mizuno S, Muraki Y, Nakatani K, et al. Immunological aspects in late phase of living donor liver transplant patients: usefulness of monitoring peripheral blood $\mathrm{CD}^{+}$ adenosine triphosphate activity. Clin Dev Immunol 2013;2013:982163.

16. Fukuda A, Imadome K, Sakamoto S, et al. Evaluation of the immune function assay in pediatric living donor liver transplantation. Pediatr Transplant 2015;19:144-52.

17. Gupta S, Mitchell JD, Markham DW, et al. Utility of the Cylex assay in cardiac transplant recipients. J Heart Lung Transplant 2008;27:817-22.

18. Yang TH, Chen YK, Xue F, et al. Influence of CYP3A5 genotypes on tacrolimus dose requirement: age and its pharmacological interaction with $\mathrm{ABCB} 1$ genetics in the Chinese paediatric liver transplantation. Int J Clin Pract Suppl 2015;(183): 53-62.

19. Kanamori M, Takahashi H, Echizen H. Developmental changes in the liver weight- and body weight-normalized clearance of theophylline, phenytoin and cyclosporine in 
children. Int J Clin Pharmacol Ther 2002;40:485-92.

20. Miloh T, Barton A, Wheeler J, et al. Immunosuppression in pediatric liver transplant recipients: Unique aspects. Liver Transpl 2017;23:244-56.

21. Miloh T. Medical management of children after liver transplantation. Curr Opin Organ Transplant 2014;19:474-9.

22. Piloni D, Magni S, Oggionni T, et al. Clinical utility of $\mathrm{CD}^{+}$function assessment (ViraCor-IBT ImmuKnow test) in lung recipients. Transpl Immunol 2016;37:35-9.

23. Jo Y, Lim J, Kim Y, et al. CD4 T-cell function assay using Cylex ImmuKnow and lymphocyte subset recovery following allogeneic hematopoietic stem cell transplantation. Transpl Immunol 2015;33:78-83.

24. Israeli M, Ben-Gal T, Yaari V, et al. Individualized immune monitoring of cardiac transplant recipients by noninvasive longitudinal cellular immunity tests. Transplantation 2010;89:968-76.

25. Bartoletti M, Vandi G, Furii F, et al. Management of immunosuppressive therapy in liver transplant recipients who develop bloodstream infection. Transpl Infect Dis 2018;20:e12930.

26. Furuichi M, Fukuda A, Sakamoto S, et al. Characteristics and Risk Factors of Late-onset Bloodstream Infection Beyond 6 Months After Liver Transplantation in Children. Pediatr Infect Dis J 2018;37:263-8.

27. Yadav SK, Saigal S, Choudhary NS, et al. Cytomegalovirus infection in living donor liver transplant recipients significantly impacts the early post-transplant outcome: A single center experience. Transpl Infect Dis 2018;20:e12905.

28. Kim SI. Bacterial infection after liver transplantation. World J Gastroenterol 2014;20:6211-20.

29. Azevedo LS, Pierrotti LC, Abdala E, et al. Cytomegalovirus infection in transplant recipients. Clinics (Sao Paulo) 2015;70:515-23.

30. Kollmann TR, Kampmann B, Mazmanian SK, et al.

Cite this article as: Xue F, Gao W, Qin T, Wu C, Luo Y, Chen J, Zhou T, Feng M, Qiu B, Zhu J, He J, Xia Q. Immune cell function assays in the diagnosis of infection in pediatric liver transplantation: an open-labeled, two center prospective cohort study. Transl Pediatr 2021;10(2):333-343. doi: 10.21037/tp-20256
Protecting the Newborn and Young Infant from Infectious Diseases: Lessons from Immune Ontogeny. Immunity 2017;46:350-63.

31. Jennewein MF, Butler AL, Alter G. Neonate-omics: Charting the Unknown Immune Response in Early Life. Cell 2018;174:1051-3.

32. Xue F, Zhang J, Han L, et al. Immune cell functional assay in monitoring of adult liver transplantation recipients with infection. Transplantation 2010;89:620-6. Retraction in: Transplantation. 2019 Aug;103(8):1737. doi: 10.1097/ TP.0000000000002824.

33. Zhou T, Xue F, Han LZ, et al. Invasive fungal infection after liver transplantation: risk factors and significance of immune cell function monitoring. J Dig Dis 2011;12:467-75.

34. Kowalski R, Post D, Schneider MC, et al. Immune cell function testing: an adjunct to therapeutic drug monitoring in transplant patient management. Clin Transplant 2003;17:77-88.

35. Cassimos DC, Liatsis M, Stogiannidou A, et al. Children with frequent infections: a proposal for a stepwise assessment and investigation of the immune system. Pediatr Allergy Immunol 2010;21:463-73.

36. Ling X, Xiong J, Liang W, et al. Can immune cell function assay identify patients at risk of infection or rejection? A meta-analysis. Transplantation 2012;93:737-43.

37. Dong JY, Yin H, Li RD, et al. The relationship between adenosine triphosphate within CD4(+) T lymphocytes and acute rejection after liver transplantation. Clin Transplant 2011;25:E292-6.

38. Ryan CM, Chaudhuri A, Concepcion W, et al. Immune cell function assay does not identify biopsy-proven pediatric renal allograft rejection or infection. Pediatr Transplant 2014;18:446-52.

39. Ashokkumar C, Soltys K, Mazariegos G, et al. Predicting Cellular Rejection With a Cell-Based Assay: Preclinical Evaluation in Children. Transplantation 2017;101:131-40. 